\title{
Providing a primary care service for psychiatric in-patients
}

\author{
AIMS AND METHOD \\ A prospective descriptive study was \\ set up to evaluate the feasibility, \\ acceptability and activity of an \\ innovative weekly primary care \\ service for patients admitted for \\ acute psychiatric care.
}

\author{
RESULTS \\ During 10 months, 36 clinics were \\ held and 123 appointments were \\ attended. Presenting complaints \\ included a wide range of acute and \\ chronic conditions, affecting all body \\ systems. As well as treating specific \\ complaints, the doctor providing \\ this service undertook considerable \\ health promotion work and gave
}

advice about patient management to junior psychiatrists.

\author{
CLINICAL IMPLICATIONS \\ It appears that there is considerable \\ need for primary care expertise \\ within an acute psychiatric unit, \\ and that a weekly clinic is a feasible \\ model of care.
}

The physical health of people with schizophrenia and other types of severe mental illness is frequently poor (Phelan et al, 2001). This association has been widely reported by researchers for decades (e.g. Harris \& Barraclough, 1998) and is repeatedly highlighted in policy documents (Department of Health, 1990, 1999) and by voluntary groups (National Schizophrenia Fellowship, 2002). In the USA, integrating a primary care service into a mental health clinic resulted in improved physical health care (Druss et al, 2001); however, the US health care system is very different from that of the UK, and there is little evidence yet to indicate how the problem can best be met within the UK system. Mental health staff report being unclear about the extent of their role in relation to physical health, and believe that service users are uninterested in their physical health (Dean et al, 2001). Service users themselves have stated that their physical health is a priority, but that they lack the relevant information and face difficulties in accessing physical health care (Friedli \& Dardis, 2002).

When someone is admitted to hospital, an opportunity arises to address acute physical health needs, and to review long-standing needs such as overdue health screening, contraception and chronic medical conditions. Current evidence suggests that this opportunity is rarely grasped. Physical examinations by psychiatric trainees are usually done badly (Rigby \& Oswald, 1987); when patients are admitted less than $75 \%$ are examined at all, and there is rarely any attempt to take a physical history (Osborn \& Warner, 1998).

The National Institute for Clinical Excellence (2002) has stated that primary care professionals are best placed to monitor the physical health of patients with schizophrenia, and should offer regular physical health checks. If patients have contact with their general practitioner, they appear to be more satisfied with the amount and type of help received (Beecroft et al, 2001). However, although many patients with a mental illness consult frequently, in routine practice opportunities for health promotion are seldom taken (Burns \& Cohen, 1998).

In an attempt to improve the physical health care received by patients admitted to an inner-city mental health unit, we decided to provide a weekly primary care service. This paper examines the feasibility and acceptability of the service, and describes its activity during the first 10 months.

\section{Description of the service}

One author (S.T.) provided a weekly 3-hour session for the 67 patients in the three acute adult psychiatry wards at Charing Cross Hospital, as well as a limited service to the older persons service. Referrals were accepted from ward doctors, nursing staff and the patients themselves. An appointment diary was kept on one of the three wards. Notes from consultations were made in the patients' files, and changes to medication were written directly on the ward drug charts. Initially, patients were seen in a treatment room separate from any of the wards; however, experience showed that it was more efficient for patients to be seen on the wards. Appointment times of 15 minutes were offered when the service was first started, but this proved inadequate for the complex needs and communication difficulties of many patients, and appointment lengths were increased to 30 minutes. After a few months the demand for the service exceeded capacity, and ward doctors were advised to give priority to patients who were homeless, not registered with a general practitioner or who had not seen one for more than 6 months, or who had been in hospital for more than 3 months. As well as seeing patients herself, S.T. offered advice to ward doctors and nurses.

\section{Method}

Data were collected for all consultations during the first 10 months of the service. After each patient contact, S.T. recorded the patient's demographic details, the presenting complaints and the physical diagnoses, outcomes and treatment. Outcomes for service (teaching and feedback) and supplementary outcomes (including health promotion and health screening) were also documented. In addition, patients who used the service over a 5-month period were approached during the week 
following the consultation and asked to participate in a semi-structured interview.

original

papers

\section{Results}

\section{Demands on service}

During the 10-month study period 36 clinics were held and 168 appointments made, $123(73.2 \%)$ of which were attended. The most common reasons for appointments being missed were patients being on leave or discharged. If patients did not attend, S.T. would frequently review their notes and give advice on their management. Sixtytwo of the patients were seen on only one occasion. Half of the appointments that the patient attended were follow-up appointments.

\section{Characteristics of patients}

During the period studied, 107 patients had appointments arranged with S.T., amounting to $22 \%$ of all patients admitted to the mental health unit during the same period. Files were not obtainable for 6 patients ( 9 appointments) and therefore only 101 patients are considered in subsequent analysis. Eighty-four patients attended their consultations, of whom 13 (15\%) were aged 65 years or more; two-thirds of the patients seen had had a physical examination performed within a week of admission, and a third of patients had had no physical examination prior to consultation. Eighty per cent of patients had had urea and electrolytes assessed and a full blood count done; $74 \%$ had had liver function tests; $69 \%$ had had thyroid function tests; $65 \%$ had had random glucose level measurement and $42 \%$ had had a cholesterol screen. Blood tests were mainly requested routinely by the attending psychiatric senior house officer. Time between the patients' date of admission to the first appointment with S.T. varied from 1 day to 1049 days, with a median of 22 days. Patients who did not attend appointments had been in hospital for a longer period before the offered appointment, compared with those who did attend (median 52 days v. 22 days), and were also less likely to have had physical investigations.

The majority of patients seen had a primary diagnosis of a severe and enduring mental illness, mainly schizophrenia (Table 1); 25 patients had a secondary psychiatric diagnosis. Substance misuse accounted for most of these, with 17 patients qualifying for a diagnosis of mental and behavioural disorder due to psychoactive substance misuse. Mental retardation (2 patients) and mood disorder (3 patients) were also present.

\section{Physical complaints}

Physical complaints with which patients presented included a wide range of acute and chronic conditions affecting all body systems (Table 2). One patient was newly diagnosed with a breast tumour following routine review, and another patient was found to have diabetes mellitus, which had not been diagnosed during a 3month admission period. A severely anaemic 47-year-old
Table 1. Characteristics of patients seen $(n=84)$

$\begin{array}{lc}\text { Gender } & \\ \quad \text { Male (\%) } & 45 \\ \text { Age, years } & 46.6 \\ \quad \text { Mean } & 17-79 \\ \quad \text { Range } & 80 \\ \text { Registered with general practitioner in the } & \\ \quad \text { community (\%) } & \\ \text { Mental Health Act 1983 status (\%) } & 57 \\ \text { Informal } & 13 \\ \text { Section 2 } & 30 \\ \text { Section 3 } & \\ \text { Diagnosis (\% of patients) } & 48 \\ \quad \text { Schizophrenia } & 23 \\ \quad \text { Bipolar affective disorder } & 18 \\ \text { Depression } & 5 \\ \text { Mental and behavioural disorders due to } & \\ \quad \text { psychoactive substance misuse (delusional } & \\ \quad \text { disorder/psychosis) } & \\ \text { Delusional disorder } & \\ \quad \text { Dementia } & \end{array}$

male patient who did not attend for his appointment, but whose notes S.T. reviewed, had an emergency blood transfusion arranged as a result. Poorly controlled hypertension and diabetes mellitus were common findings.

\section{Interventions}

New medication for physical illness was prescribed in 66 consultations and existing medication altered in a further 8 consultations. 'Watchful waiting' was adopted following 49 consultations. Twenty patients required referral to other specialists as a result of the primary care consultation, and 8 needed follow-up appointments with S.T. Almost all patients seen $(97 \%)$ received health promotion (Table 3). Health promotion advice or education relating to the patient's specific illness profile or underlying risk factors was given by S.T. in 54 consultations, for example dietary advice for patients with diabetes mellitus. Further activities included reassurance of patients with anxieties related to physical health, liaison with other professionals and advice on exercise (61 occasions). Feedback and education of nursing and medical staff about specific patients and their medical conditions was provided on 64 occasions.

\section{Patients' perspectives}

Eighteen of 45 patients seen by S.T. over a 5 -month period were interviewed. Of the 27 patients not interviewed, approximately a third refused, a third were on leave or had been discharged, and a third were not able to consent to interview as a result of their mental illness. Three-quarters of those interviewed, when asked, felt that their psychiatric teams took their physical needs seriously. When asked about their experience of the service, all patients were satisfied with the care provided by S.T., who was variously described as professional, kind 
Table 2. Physical diagnoses documented during consultation

Diagnosis

Infectious and parasitic diseases
Fungal infections
Scabies/lice
Gastroenteritis
Neoplasms
Myelomatosis

Diseases of the blood-forming organs and certain

disorders involving the immune mechanism

Anaemia

Endocrine, nutritional and metabolic diseases

Diabetes mellitus

Hypercholesterolaemia

Obesity

Premature menopause

Diseases of the eye and adnexa

Glaucoma

Conjunctivitis

Visual disturbance

Diseases of the ear and mastoid process

Earwax

Ear pain

Otitis externa

Tinnitus

Diseases of the circulatory system

Hypertension

Congestive cardiac failure

Claudication

Chronic obstructive airways disease

Haemorrhoids

Diseases of the respiratory system

Respiratory tract infection

Asthma

Diseases of the digestive system

Oral diseases

Rectal bleeding

Gastritis

Diseases of the skin and subcutaneous tissue

Skin infection/rash

Eczema

Nail disorder

Diseases of the musculoskeletal system and connective tissue

Limb pain/swelling

Neck/back/sciatic pain

Arthritis

Diseases of the genitourinary system

Breast lump/pain

Impotence

Urinary tract infection

Amenorrhoea

Vaginal pain

Dysmenorrhoea

Orchitis

Symptoms, signs and abnormal clinical and laboratory

findings not elsewhere classified

Abdominal pain/distension

Urinary incontinence, hesitancy

Pyrexia of unknown origin

Snoring

Mass on scalp

Vomiting

Tiredness

Dizziness

Shortness of breath
Table 2. (continued)

\begin{tabular}{lr} 
Diagnosis & $n^{1}$ \\
\hline $\begin{array}{l}\text { Injury, poisoning and certain other consequences of } \\
\text { external causes }\end{array}$ & 1 \\
Leg injury & 1 \\
Factors influencing health status and contact with health & 18 \\
services & 7 \\
Family planning & 4 \\
Screening for sexually transmitted diseases & 3 \\
Routine medical review & 2 \\
Hearing check & 1 \\
Smoking & 1 \\
Anorexia physical review & \\
1. Patients seen on more than one occasion might have had same diagnosis \\
documented more than once, and many patients had multiple physical \\
diagnoses.
\end{tabular}

original

papers

and understanding. One patient was anxious about having to see a doctor other than her psychiatrist.

When asked about who they would prefer to see, half the patients stated that they would prefer review by the ward general practitioner during their in-patient stay to seeing their own general practitioner, mainly due to ease of access and easy communication with the ward doctor. Four patients preferred to see their own doctor, usually citing familiarity with their health as the reason. Five patients had no preference. Only one patient raised concern about seeing the ward general practitioner; this person wanted the number of professionals involved in providing care to be limited. Five patients made suggestions for enhanced service, in the form of more clinics per week and more information for new patients regarding access to the service. Another suggested combined assessment by the ward general practitioner and the psychiatric team.

\section{Discussion}

Having a primary care service for an acute psychiatric unit appears to help meet the numerous acute and long-

Table 3. Health promotion interventions during consultations

\begin{tabular}{lr} 
Intervention & $n$ \\
\hline Smoking cessation advice & 74 \\
Exercise advice & 31 \\
Dietary advice & 47 \\
Sexual health promotion & 32 \\
Alcohol advice & 30 \\
Drugs advice & 10 \\
Relationship/family issues & 26 \\
Screening, e.g. blood pressure monitoring, cervical & 63 \\
smear, cholesterol testing & \\
Family planning & 29 \\
Other & 4 \\
Total & 346
\end{tabular}


standing physical health needs of patients. Previous work by Fisher \& Roberts (1998) has highlighted the important role for a primary care doctor among long-stay patients. Our study suggests that patients in acute psychiatric wards can also benefit from primary care expertise. It also suggests that it is feasible to establish a primary care service, and that such a service usefully supplements the physical health care already provided and is welcomed by patients. Potential problems considered when the service was established did not really materialise. Some duplication of work occurred initially when patients were referred to specialist on-call services as well as to the primary care service, but feedback to staff minimised such events. An initial concern of staff was that the service would introduce an extra layer of complexity in the care of patients. In practice, this did not appear to be a problem.

This study only describes what was done in one acute unit, and it cannot be assumed that similar services would be successful elsewhere under routine conditions. While we were monitoring activity, changes to appointments and service criteria were introduced to control the demand that could not always be met. No health impact assessment has been done, but it would appear that this service does fill a gap in terms of physical health care provision for patients with severe mental illness. The patient interviews were limited in number, and did not necessarily capture the views of those who did not use the service.

It has often been argued that psychiatrists are doctors, and should themselves be expected to improve the physical health care that is currently offered to patients, but this rarely seems to happen. Our work has confirmed previous research highlighting the inadequate physical health care routinely received on psychiatric wards, and provides one model of care to try to improve matters. It further suggests that greater efforts may be needed to engage certain patients, as those who were never seen had longer durations of admission before their appointments and lower rates of physical investigations. Further research is needed to assess the effectiveness of such services in bringing about health improvements. In the meantime, after this 12-month pilot study, our local primary care trust has agreed to continue funding the primary care service at Charing Cross Hospital, where a new general practitioner is settling in.

\section{Acknowledgements}

The authors would like to thank Trevor Farmer, Linda Massie, Miranda Mead and Dr Frank Kelly, who made this work possible.

\section{Declaration of interest}

None.

\section{References}

BEECROFT, N., BECKER, T., GRIFFITHS G., et al (2001) Physical health care for people with severe mental illness: the role of the general practitioner. Journa of Mental Health, 10,53-61.

BURNS, T. \& COHEN, A. (1998) Item of Service payments for general practitioner care of severely mentally ill patients: does the money matter? British Journal of General Practice, $\mathbf{4 8}$ 1415-1416.

DEAN, J.,TODD, G., MORROW, $H_{\text {., et al }}$ (2001) 'Mum, I used to be good looking look at me now'. The physical health needs of adults with mental health problems: the perspective of users, carers and front-line staff. Internationa Journal of Mental Health Promotion, 3 , 16-24.

DEPARTMENT OF HEALTH (1990) The Care Programme Approach. Circular HC(90)23/LASSL(90)11. London: Department of Health

DEPARTMENT OF HEALTH (1999) A National Service Framework for Mental Health. London: HMSO.

DRUSS, B. G., ROHRBAUGH, R. M. LEVINSON, C. M., et al (2001) Integrated medical care for patients with a serious psychiatric illness. Archives of General Psychiatry, $\mathbf{5 8}$ 861-868.

FISHER, N. \& ROBERTS, J. (1998) Primary health care service for long-stay psychiatric patients. Psychiatric Bulletin, 22, 610-612.

FRIEDLI, L. \& DARDIS, C. (2002) Not all in the mind: mental health service user perspectives on physical health. Journal of Mental Health Promotion, 1, 36-46.

HARRIS, E. C. \& BARRACLOUGH, B. (1998) Excess mortality of mental disorder. British Journal of Psychiatry, 173, 11-53.

NATIONAL INSTITUTE FOR CLINICAL EXCELLENCE (2002) Core Interventions in theTreatment and Management of Schizophrenia in Primary and Secondary Care. London: NICE.

NATIONAL SCHIZOPHRENIA

FELLOWSHIP(2002) Doesn't It Make You Sick? London: National Schizophrenia Fellowship.

OSBORN, D. \& WARNER, J. (1998) Assessing the physical health of psychiatric patients. Psychiatric Bulletin, 22, 695-697.

PHELAN, M., STRADINS, L. \& MORRISON, S. (2001) Physical health of people with severe mental illness. BMJ, $322,443-444$

RIGBY, J. C. \& OSWALD, A. G. (1987) An evaluation of the performing and recording of physical examinations by psychiatric trainees. British Journal of Psychiatry, 150, 533-535.
Enone Welthagen Specialist Registrar in Psychiatry, South West London and St George's Mental Health NHS Trust, Springfield Hospital, 61 Glenburnie Road, London SW17 7DJ, SarahTalbot General Practitioner, The Surgery, 82 Lillie Road, Fulham, London SW6 1TN, Oliver Harrison Senior House Officer in Psychiatry, Charing Cross Hospital, Fulham Palace Road, London W6 8AF, *Michael Phelan Consultant Psychiatrist, West London Mental Health Trust, Gloucester House, 194 Hammersmith Road, London W6 8BS 